Published in final edited form as:

Dallabernardina, P., Schuhmacher, F., Seeberger, P. H., \& Pfrengle, F. (2017). Mixed-Linkage

Glucan Oligosaccharides Produced by Automated Glycan Assembly Serve as Tools to

Determine the Substrate Specificity of Lichenase. Chemistry - A European Journal, 23(13),

3191-3196. doi:10.1002/chem.201605479.

\title{
Mixed-Linkage Glucan Oligosaccharides Produced by Automated Glycan Assembly Serve as Tools to Determine the Substrate Specificity of Lichenase
}

Pietro Dallabernardina, Frank Schuhmacher, Peter H. Seeberger and Fabian Pfrengle

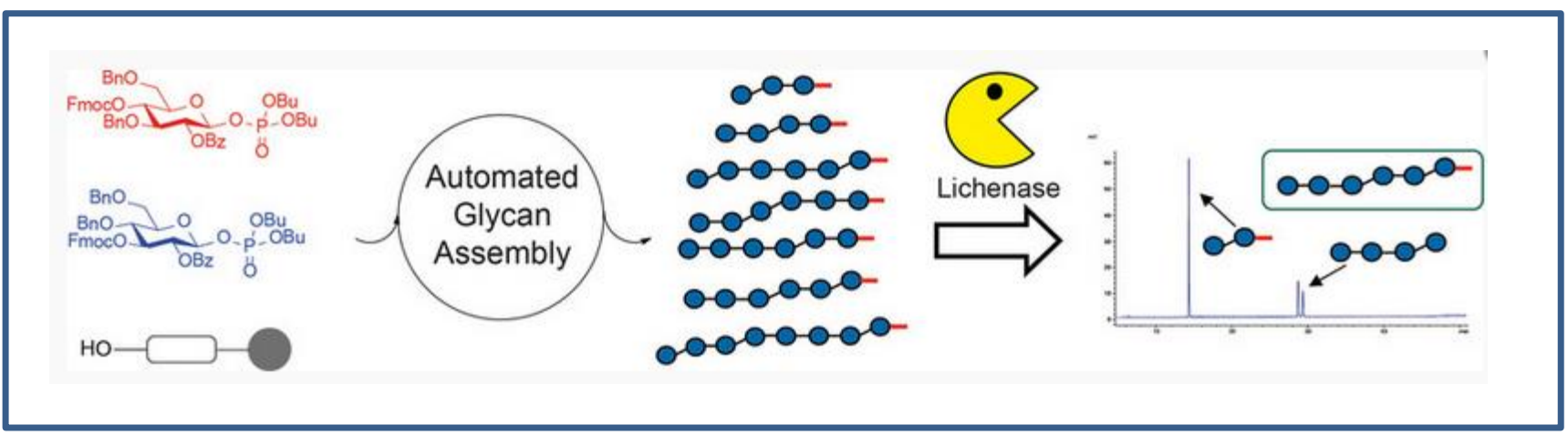

Assembly and deconstruction: The automated glycan assembly of mixed-linkage glucan oligosaccharides as biochemical tools for analyzing the substrate specificities of lichenase and other mixed-linkage $\beta$-glucanases is reported (see scheme).

This article may be used for non-commercial purposes in accordance with Wiley Terms and Conditions for Self-Archiving. 


\title{
Mixed-Linkage Glucan Oligosaccharides Produced by Automated Glycan Assembly Serve as Tools to Determine the Substrate Specificity of Lichenase
}

\author{
Pietro Dallabernardina, Frank Schuhmacher, Peter H. Seeberger and Fabian Pfrengle*
}

\begin{abstract}
The mixed-linkage $(1 \rightarrow 3),(1 \rightarrow 4)-D-$ glucan $(M L G)$ specific glycosyl hydrolase lichenase is an important biochemical tool for the structural characterization of MLGs. It holds potential for application in the brewery, animal feed, and biofuel industries. Several defined MLG oligosaccharides obtained by automated glycan assembly are used to analyze the substrate specificities of Bacillus subtilis lichenase. Two glucose building blocks (BBs), equipped with a temporary Fmoc protecting group in the C-3 or C-4 position, served to assemble different oligosaccharides using an automated oligosaccharide synthesizer. Light-induced cleavage of the glycan products from the solid support followed by global deprotection provided seven MLG oligosaccharides of different length and connectivity. After incubation of the MLG oligosaccharides with lichenase, the digestion products were analyzed by HPLC-MS. These digestion experiments provided insights into the enzyme's active site that is in line with other recent evidence suggesting that the substrate specificity of lichenases has to be reconsidered. These results demonstrate that synthetic MLG oligosaccharides are useful tools to analyse mixed-linkage $\beta$-glucanases.
\end{abstract}

\section{Introduction}

Mixed-linkage glucan (MLG) is a hemicellulosic polysaccharide from the cell wall of grasses and cereals. ${ }^{[1]}$ As an important component of dietary fiber, MLG exhibits beneficial effects on human health, including reduction of colorectal cancer risk, ${ }^{[2]}$ lowering of blood cholesterol, and regulation of blood glucose levels for diabetes management. ${ }^{[3]}$ MLGs are considered as attractive additives for the manufacturing of lowfat food. ${ }^{[4]}$

The structure of MLG is represented by an unbranched glucan chain composed of short stretches of $\beta$-1,4-linked oligosaccharides connected through $\beta$-1,3-linkages. ${ }^{[5]}$ These cello-oligosaccharide sequences typically consist of three to five

["] Pietro Dallabernardina, Frank Schuhmacher, Prof. Dr. Peter H Seeberger, Dr. Fabian Pfrengle

Department of Biomolecular Systems, Max-Planck-Institute of Colloids and Interfaces,

Am Mühlenberg 1, 14476 Potsdam, Germany

E-mail: Fabian.Pfrengle@mpikg.mpg.de

and

Freie Universität Berlin, Institute of Chemistry and Biochemistry, Arnimallee 22, 14195 Berlin, Germany.

Supporting information for this article is given via a link at the end of the document.

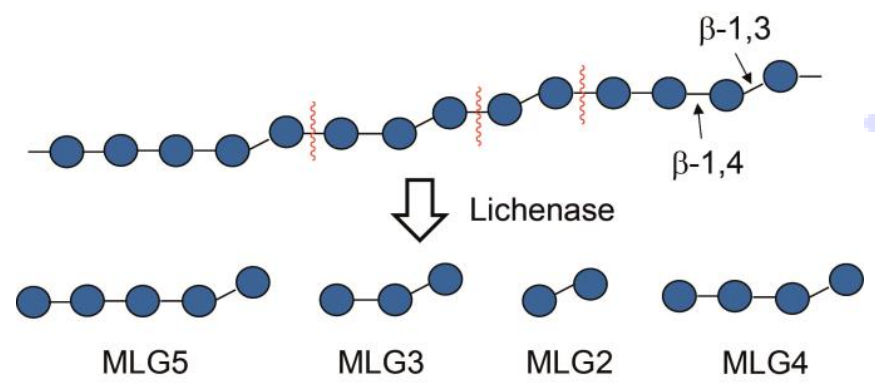

Figure 1. Schematic representation of mixed-linkage glucan polysaccharides and the oligosaccharide fragments obtained after lichenase treatment.

residues, but also shorter and longer stretches may be found. ${ }^{[6]}$ The 1,3-linkages in the 1,4-linked glucan chain form molecular kinks that prevent an intermolecular alignment into microfibrils as observed in the case of cellulose. Instead, the limited intermolecular interactions result in the formation of a gel-like material which not only provides the cell wall with strength, but also flexibility and other important characteristics of cellular function. ${ }^{[7]}$

Besides holding enormous potential as biocatalysts for the brewery and animal feed industries to produce high quality brews and better digestible feed, ${ }^{[8]}$ MLG-degrading enzymes are essential tools for the structural characterization of MLGs. ${ }^{[9]}$ The MLG-specific hydrolase lichenase cleaves every 1,4-linkage following a 1,3-linkage in a mixed-linkage glucan backbone (Figure 1). ${ }^{[10]}$ The resulting oligosaccharide fragments are usually represented as MLGX, with $X$ describing the number of glucose units contained. Recently, the inevitability of the described lichenase specificity was questioned when an intact hexasaccharide composed of a MLG2 and a MLG4 fragment was obtained after digestion of MLG with lichenase. ${ }^{[6]}$ This discovery has obvious implications on the reported structure of MLGs, as their subunit distribution is mostly derived from the analysis of lichenase digestion products.

Synthetic MLG-oligosaccharides of varying connectivity would permit a simple LC-MS analysis of their digestion products after hydrolysis, providing a toolkit for the determination of the substrate specificities of lichanase and other MLG endoglucanases. Automated glycan assembly ${ }^{[11]}$ provides a powerful platform for the synthesis of such collections of structurally related glycans. ${ }^{[12]}$ We report here the automated synthesis of seven MLG oligosaccharides and their application in the characterization of the active site of lichenase. 


\section{Results and Discussion}

For the synthesis of the MLG oligosaccharides, two glucose building blocks (BBs 1 and 2) were employed that, in similar form, already provided good results in the automated glycan assembly of either exclusively $\beta-1,3$ - or $\beta-1,4$-linked glucan oligosaccharides (Scheme 1). ${ }^{[12 b, 13]}$ Fmoc was chosen as the temporary protecting group for chain elongation because it is smoothly cleaved during automated assembly using amine bases. Permanent protection was realized with $\mathrm{Bz}$ esters in the C2-position to ensure $\beta$-selectivity in the glycosylation reactions. Bn ethers that are stable during assembly, but smoothly cleaved by hydrogenation during global deprotection, were installed in all other positions. Phosphate served as the leaving group since glycosyl phosphates provided the best glycosylation efficiencies in the automated assembly of long oligosaccharide chains previously. ${ }^{[12]}$ In order to determine the amounts of glycosyl donors $\mathbf{1}$ and $\mathbf{2}$ required for the individual glycosylation steps in the automated glycan assembly of MLG oligosaccharides, trisaccharide $\mathbf{4}$ was chosen as a simple test substrate (Figure 2). After TMSOTf-promoted glycosylation of linker-functionalized resin $3^{[14]}$ using one cycle of 3.7 equiv 1 , the Fmoc-group was cleaved using $\mathrm{NEt}_{3}$ and the resulting free $4-\mathrm{OH}$ was glycosylated with 3.7 equiv 2. Fmoc was cleaved and another glycosylation with 3.7 equiv 1 was performed. After removal of the terminal Fmoc-group, the reaction products were cleaved from the resin in a continuous flow photoreactor and analyzed by HPLC. Besides the desired product $\mathbf{4}$, a side product was formed that was identified as a disaccharide deletion sequence. It was not possible to deduce from the HPLC-analysis which glycosylation reaction did not reach completion (Figure $2 \mathrm{~b}$ ).

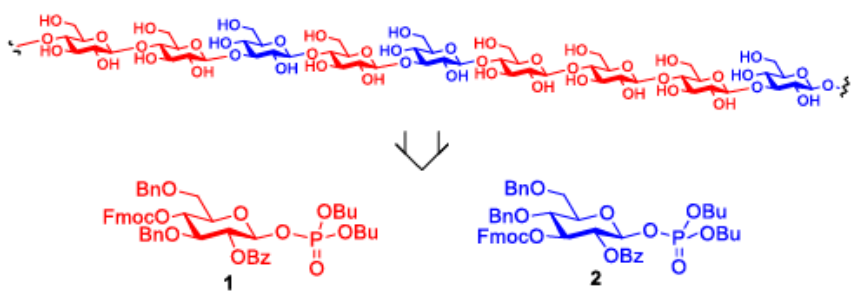

Scheme 1. Representative chemical structure of mixed-linkage glucan polysaccharides and he building blocks required for the automated glycan assembly of oligosaccharide fragments.

Since we knew from previous studies that one cycle of 3.7 equiv glycosyl donor is sufficient for the synthesis of cellooligosaccharides using BB 1, two possible reasons for the incomplete reaction remained: either BB 2 is less reactive than BB 1 and the second glycosylation was inefficient, or the C-3 hydroxyl group in the third glycosylation reaction exhibits reduced nucleophilicity compared to the C-4 hydroxyl in the second glycosylation. To test the first option we repeated the synthesis, but used two cycles of 3.7 equiv BB 2. However, HPLC-analysis of the products revealed the same amount of deletion sequence as before (Figure 2b). Only when we used two cycles of 3.7 equiv BB 1 in the third glycosylation, we were able to obtain full conversion. Thus, we performed one glycosylation cycle for the formation of $\beta$-1,4-linkages and two cycles for $\beta-1,3$-linkages in all later syntheses.

a)
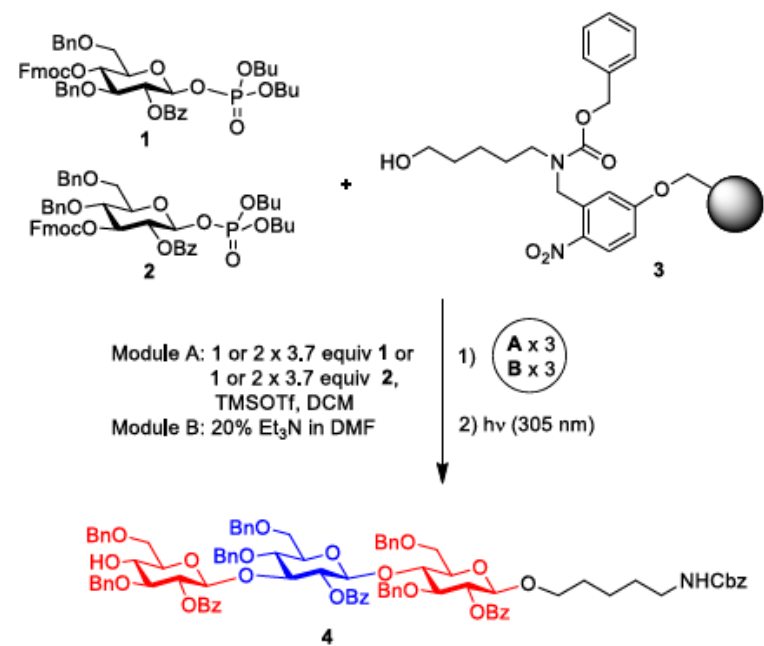

b)
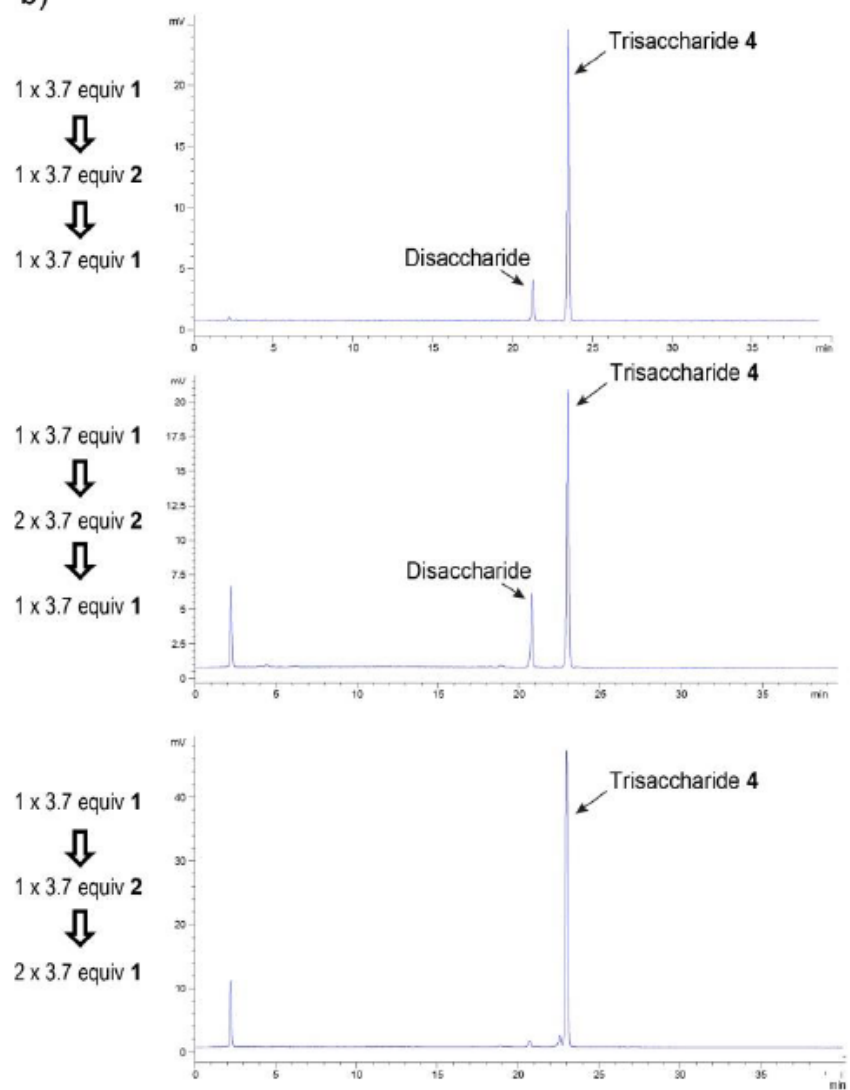

Figure 2. Optimiza ion of the automated glycan assembly process towards mixed-linkage glucan oligosaccharides. a) Automated glycan assembly of trisaccharide 4 . Reagents and conditions: 1 or $2 \times 3.7$ equiv. BB 1, TMSOTf, $\mathrm{CH}_{2} \mathrm{Cl}_{2},-30{ }^{\circ} \mathrm{C}(5 \mathrm{~min}) \rightarrow-15^{\circ} \mathrm{C}(30 \mathrm{~min})$ (Module $\left.\mathrm{A}\right) ; 3$ cycles of $20 \% \mathrm{NEt}_{3}$ in DMF, $25^{\circ} \mathrm{C}$ (5 min) (Module B). b) HPLC analysis (ELSD trace) of the crude products after the different automated assembly processes. 

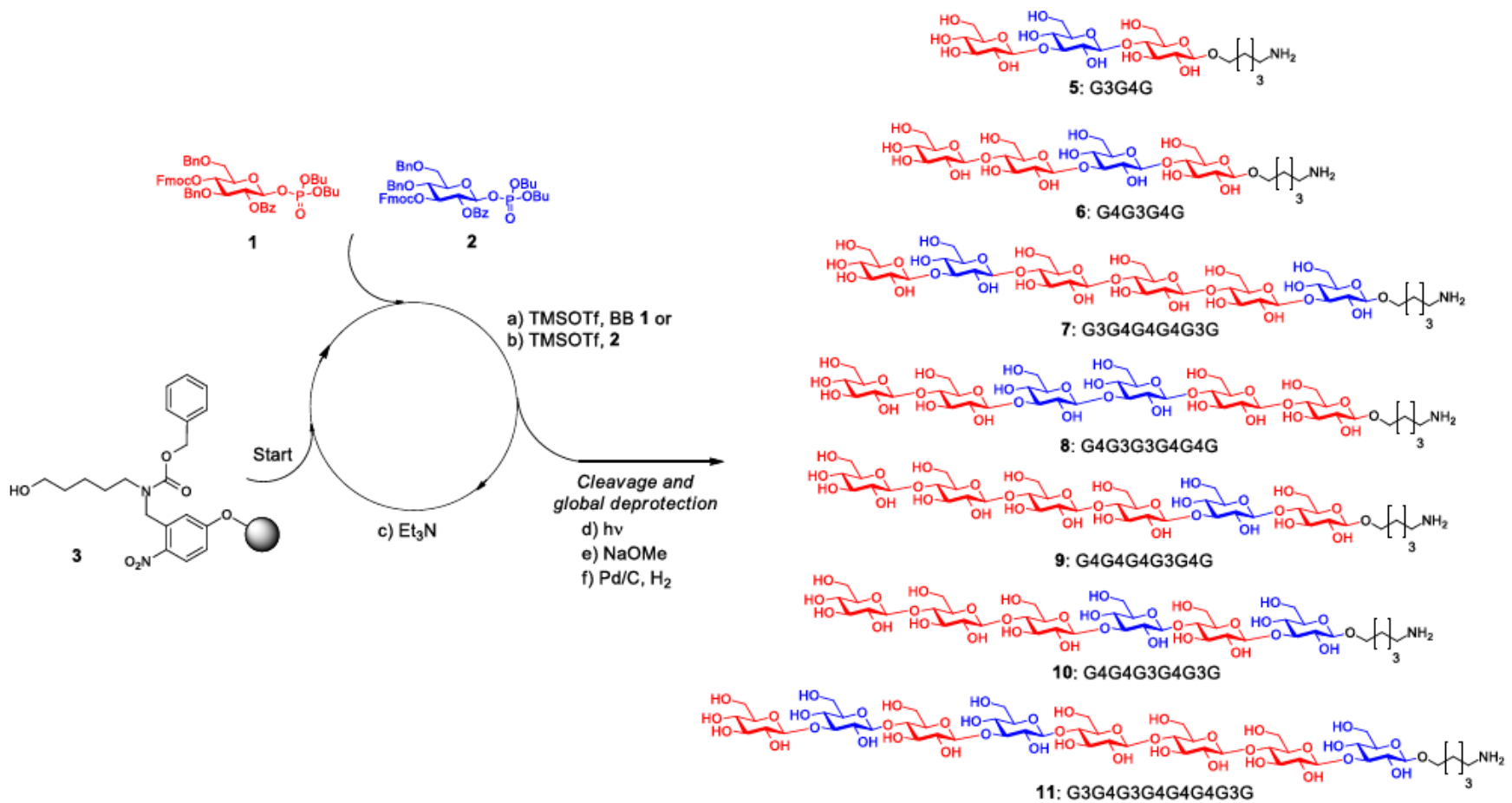

Scheme 2. Automated glycan assembly of MLG oligosaccharides. Reagents and conditions: (a) 1 or $2 \times 3.7$ equiv $\mathrm{BB} 1, \mathrm{TMSOTf}^{\circ} \mathrm{CH}_{2} \mathrm{Cl}{ }_{2},-30{ }^{\circ} \mathrm{C}(5$ min) $\rightarrow$ $-15^{\circ} \mathrm{C}$ (30 min) (Module A); b) 1 or $2 \times 3.7$ equiv BB 2. TMSOTf, $\mathrm{CH}_{2} \mathrm{Cl}_{2},-30{ }^{\circ} \mathrm{C}(5 \mathrm{~min}) \rightarrow-15^{\circ} \mathrm{C}$ (30 min) (Module A); (c) $3 \mathrm{cycles}$ of $20 \% \mathrm{NEt}$ in DMF, $25^{\circ} \mathrm{C}$ (5 min) (Module B); (d) $\mathrm{CH}_{2} \mathrm{Cl}_{2}$, hv (305 nm); (e) NaOMe, THF/MeOH, 12 h; (f) $\mathrm{H}_{2}, \mathrm{Pd} / \mathrm{C}$, EtOAc/MeOH/H $\mathrm{H}_{2} \mathrm{O} / \mathrm{HOAc}, 12 \mathrm{~h} .5$ : $13 \%$; 6: 26\%; $7: 12 \%$; 8: 12\%; 9: 34\%; 10: $18 \% ; 11: 23 \%$ (yields are based on resin loading). The letter code below the structures refers to a common nomenclature of MLG oligosaccharides. ${ }^{[6]}$
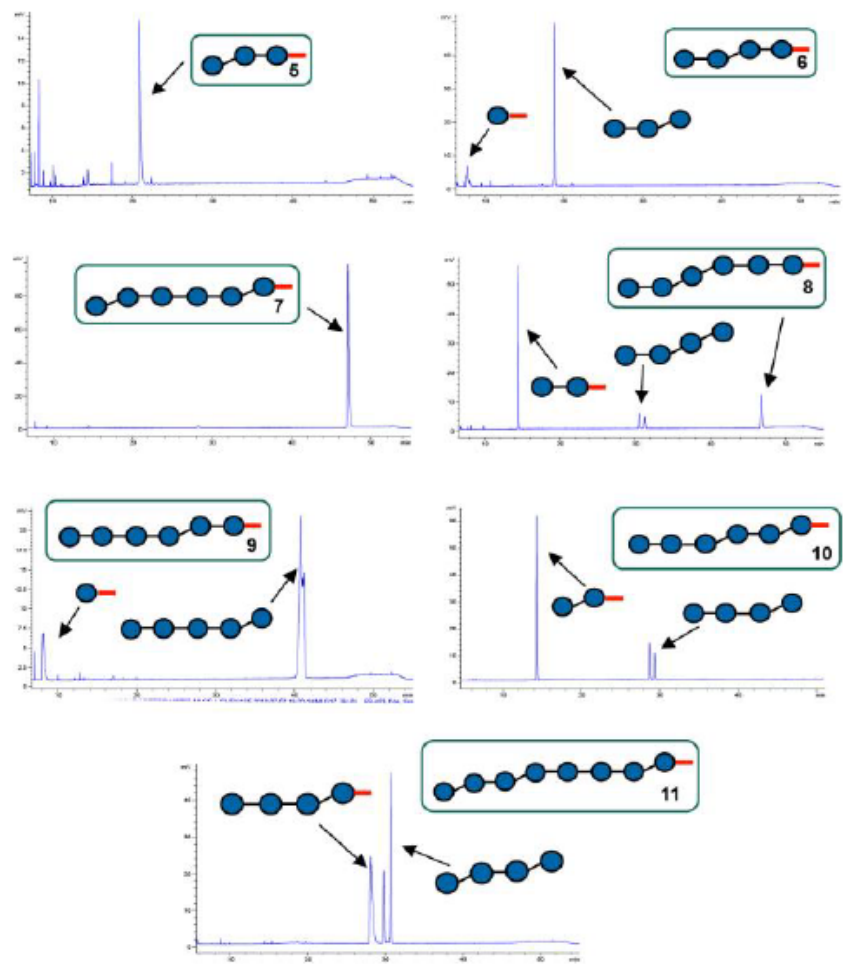

Figure 3: Digestion of synthetic MLG oligosaccharides by lichenase and HPLC-MS analysis of the reaction products. Peaks are annotated with MLG fragments with aminopentenyl linker or with free reducing end (with or without red bar). Note that the $\alpha$ - and $\beta$-forms of the fragments with free-reducing end usually elute as separate peaks.
Using the optimized glycosylation conditions, we synthesized a series of MLG oligosaccharides (5-11) that were obtained after global deprotection in $12-34 \%$ yield based on resin loading (Scheme 2). In order to enable a comprehensive investigation of the substrate specificity of lichenase, the oligosaccharides were designed to cover different distances between the 1,3-linkages and different numbers of 1,4-linked glucose residues at the nonreducing end. The prepared collection of MLG oligosaccharides was incubated with lichenase from Bacillus subtilis (Glycosyl Hydrolase family 16), and after three hours the resulting digestion products were analyzed by HPLC coupled to a mass spectrometer and an Evaporative Light Scattering Detector (ELSD) (Figure 3).

Compounds 6, 9 and $\mathbf{1 0}$ were hydrolyzed according to the general observation that lichenase cleaves the 1,4-linkage following a 1,3-linkage (Figure 3). However, hexasaccharide 7 , which represents the "lichenase-resistent" structure reported by Simmons et al. ${ }^{[6]}$ was not hydrolyzed. Similarly, in octasaccharide $\mathbf{1 1}$ only one of the two glycosidic bonds following a 1,3-linkage was hydrolyzed. The fact that the MLG2 moiety at the non-reducing end of $\mathbf{1 1}$ was not cleaved confirms the hypothesis that lichenase is not able to release MLG2 fragments from the non-reducing end of MLG oligo- or polysaccharides. ${ }^{[6]}$ The unnatural structure 8 containing two consecutive 1,3linkages was only partially hydrolyzed.

To investigate the importance of the -3 subsite relative to the site of hydrolysis for substrate recognition, we determined the relative rate differences between the hydrolysis of compounds $\mathbf{7}$, $\mathbf{8}$ and $\mathbf{9}$ in a time course experiment. No significant hydrolysis 
was observed after incubating compound 7 with lichenase for 24 hours (Figure 4a). In contrast, compound 8 having a $\beta-1,3$-linked glucose residue in the -3 subsite was slowly hydrolyzed, demonstrating positive interactions of the glucose residue with the enzyme. When the -3 subsite was occupied with a $\beta-1,4-$ linked glucose residue such as in compound 9 , hydrolysis was complete within 30 minutes. Thus, occupation and correct linkage-type of the -3 subsite is key for efficient digestion (Figure $4 b)$, confirming the observation that no MLG2 fragments are released from the non-reducing end of MLG oligo- and polysaccharides.

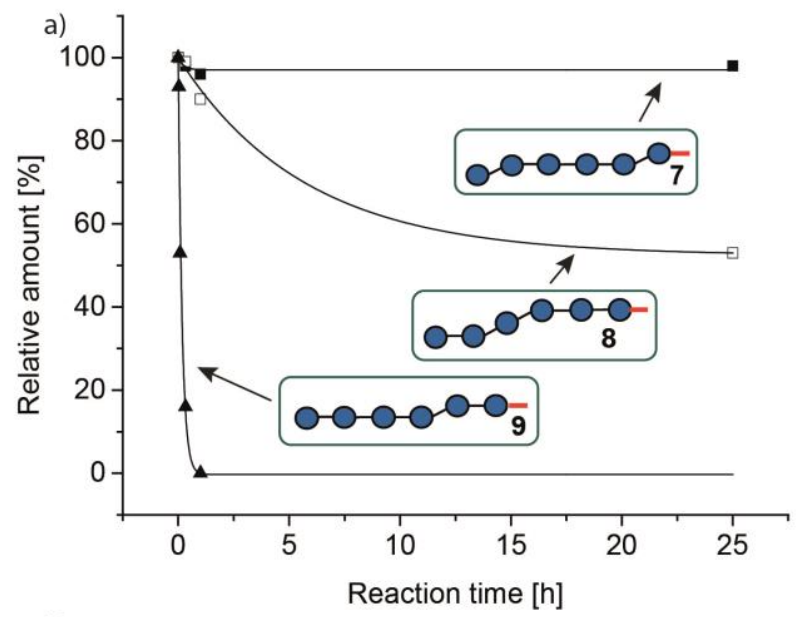

b)

7

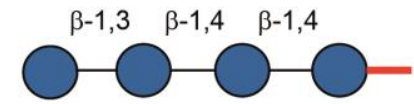

8

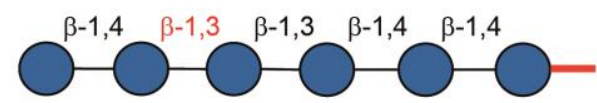

9

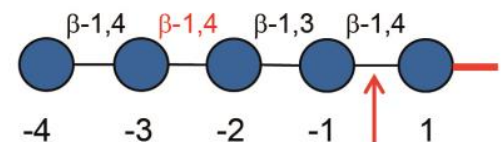

2

Figure 4: Comparison of the lichenase cleavage rates between oligosaccharides 7,8 , and 9. a) Time-course experiment showing the digestion of the substrates over a period of $24 \mathrm{~h}$. The structure of the substrates is indicated by boxes. b) Schema ic representation of oligosaccharides 7-9 and the subsites occupied during hydrolysis. The linker is indicated by a red bar and the cleavage site is denoted by a red arrow.

\section{Conclusions}

Two differentially protected glucose BBs suffice for the automated glycan assembly of a set of tailor-made natural and unnatural MLG oligosaccharides. These synthetic oligosaccharides were key to determining the substrate specificity of the MLG-degrading enzyme lichenase. Incubation of the glycans with lichenase resulted in digestion products that were analyzed by HPLC-MS. Simple end-point measurements confirmed recent reconsiderations ${ }^{[6]}$ concerning the substrate specificity of lichenase. As a consequence, structural analyses of MLG polysaccharides have to be reconsidered. The fact that lichenase does not release disaccharides from the non-reducing end of MLG oligo- and polysaccharides, highlights the importance of the -3 subsite relative to the site of hydrolysis for substrate recognition. Further information on the binding requirements in the -3 subsite were obtained by comparing the hydrolysis rates of three MLG oligosaccharides having either no, a $\beta-1,3$-linked, or a $\beta-1,4$-linked glucose residue in the -3 subsite in a time-course experiment. The MLG oligosaccharides we describe provide a convenient means for determining the substrate specificities of newly discovered mixed-linkage $\beta$ glucanases. $^{[15]}$

\section{Experimental Section}

\section{Synthesizer modules and conditions}

The linker-functionalized resin 3 (16.6-17.9 $\mu \mathrm{mol}$ of hydroxyl groups) was placed in the reaction vessel of the automated oligosaccharide synthesizer and swollen for at least $30 \mathrm{~min}$ in DCM. Before every synthesis the resin was washed with DMF, THF and DCM. Subsequently the glycosylation (Module A) and deprotection (Module B) steps were performed. Mixing of the components was accomplished by bubbling Argon through the reaction mixture.

\section{Module A: Glycosylation with glycosyl phosphates}

The resin (16.6-17.9 $\mu \mathrm{mol}$ of hydroxyl groups) was swollen in DCM (2 $\mathrm{mL}$ ) and the temperature of the reaction vessel was adjusted to $-30{ }^{\circ} \mathrm{C}$. Prior to the glycosylation reaction the resin was washed with TMSOTf in DCM and then DCM only. For the glycosylation reaction the DCM was drained and a solution of phosphate BB (3.7 equiv in $1 \mathrm{~mL} D C M$ ) was delivered to the reaction vessel. After the set temperature was reached, the reaction was started by the addition of TMSOTf in DCM (3.7 equiv in $1 \mathrm{~mL}$ DCM). The glycosylation was performed for $5 \mathrm{~min}$ at $-30{ }^{\circ} \mathrm{C}$ and then at $-15^{\circ} \mathrm{C}$ for 30 minutes. Subsequently the solution was drained and the resin was washed three times with DCM. The whole procedure was performed once or twice. Afterwards the resin was washed three times with DCM at $25^{\circ} \mathrm{C}$.

\section{Module B: Fmoc deprotection.}

The resin was washed with DMF, swollen in $2 \mathrm{~mL}$ DMF and the temperature of the reaction vessel was adjusted to $25{ }^{\circ} \mathrm{C}$. Prior to the deprotection step the DMF was drained and the resin was washed with DMF three times. For Fmoc deprotection $2 \mathrm{~mL}$ of a solution of $20 \% \mathrm{Et}_{3} \mathrm{~N}$ in DMF was delivered to the reaction vessel. After 5 min the solution was drained and the whole procedure was repeated another two times. After Fmoc deprotection was complete the resin was washed with DMF, THF and DCM.

\section{Cleavage from the solid support}

After assembly of the oligosaccharides cleavage from the solid support was accomplished by modification of a previously published protocol, ${ }^{[14]}$ using the Vapourtec E-Series UV-150 photoreactor Flow Chemistry System. The medium pressure metal halide lamp is filtered using the commercially available red filter. The resin, suspended in DCM, was loaded into a plastic syringe. The suspension was then pumped using a syringe pump (PHD2000, Harvard Aparatus) at $1 \mathrm{~mL} / \mathrm{min}$ through a 10 $\mathrm{mL}$ reactor, constructed of $1 / 8$ inch o.d. FEP tubing. The total volume within the photoreactor was $9 \mathrm{~mL}$. The temperature of the photoreactor was maintained at $20{ }^{\circ} \mathrm{C}$ and the lamp power was $80 \%$. The exiting flow was deposited in a $10 \mathrm{~mL}$ syringe containing a filter, with a collection flask beneath the syringe. 


\section{Global deprotection}

The protected oligosaccharide was dissolved in THF $(3 \mathrm{~mL})$ and $\mathrm{NaOMe}$ $(0.5 \mathrm{M}$ in $\mathrm{MeOH}, 0.5 \mathrm{~mL}$ or $1 \mathrm{~mL}$ ) was added. The reaction mixture was stirred overnight and subsequently neutralized by addition of prewashed Amberlite IR-120 resin. The resin was filtered off and the solvents were removed in vacuo. The crude product was purified by preparative HPLC, dissolved in a mixture of EtOAc/MeOH/AcOH/ $\mathrm{H}_{2} \mathrm{O}(4: 2: 2: 1,3 \mathrm{~mL})$, and the resulting solution was added to a round-bottom flask containing $\mathrm{Pd} / \mathrm{C}$ (10\% Pd, 10-20 mg). The suspension was saturated with $\mathrm{H}_{2}$ for 30 min and stirred under an $\mathrm{H}_{2}$-atmosphere overnight. After filtration of the reaction mixture through a syringe filter the solvents were evaporated to provide the fully deprotected oligosaccharide.

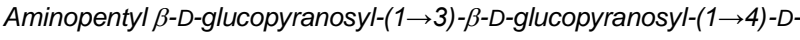 glucopyranose (5)}

The synthesizer modules were applied as follows: $\mathrm{A}(\mathbf{1}, 1$ cycle)-B-A(2, 2 cycles)-B-A(1, 1 cycle). The resulting trisaccharide was purified using normal phase HPLC (YMC Diol column). After methanolysis of the benzoyl esters the semi-protected trisaccharide was purified using reversed phase HPLC (C5 column) and subjected to hydrogenolysis, providing $5(1.3 \mathrm{mg})$ in $13 \%$ yield based on resin loading. ${ }^{1} \mathrm{H}$ NMR (600 $\left.\mathrm{MHz}, \mathrm{D}_{2} \mathrm{O}\right): \delta 4.76(\mathrm{~d}, J=7.9 \mathrm{~Hz}, 1 \mathrm{H}), 4.56(\mathrm{~d}, J=8.0 \mathrm{~Hz}, 1 \mathrm{H}), 4.50(\mathrm{~d}, J$ $=8.0 \mathrm{~Hz}, 1 \mathrm{H}), 4.08-3.26(\mathrm{~m}, 2 \mathrm{H}), 2.98(\mathrm{t}, J=7.6 \mathrm{~Hz}, 2 \mathrm{H}), 1.72-1.64(\mathrm{~m}$, $4 \mathrm{H}), 1.50-1.43(\mathrm{~m}, 2 \mathrm{H}) \mathrm{ppm} .{ }^{13} \mathrm{C}$ NMR $\left(600 \mathrm{MHz}, \mathrm{D}_{2} \mathrm{O}\right): \delta 100.2,99.8$, $99.5,81.5,76.1,73.5,73.0,72.2,71.9,70.9,70.4,67.6,67.0,65.5,58.0$, 57.5, 36.9, 25.7, $19.6 \mathrm{ppm}$. ESI-HRMS: $\mathrm{m} / \mathrm{z}[\mathrm{M}+\mathrm{H}]^{+}$calcd. for $\mathrm{C}_{23} \mathrm{H}_{44} \mathrm{NO}_{16}$ : 590.2660; found 590.2661

Aminopentyl $\quad \beta$-D-glucopyranosyl-(1 $\rightarrow 4)-\beta$-D-glucopyranosyl-(1 $\rightarrow 3)-\beta$ - $D$ glucopyranosyl-(1 $\rightarrow 4)$-D-glucopyranose $(6)$

The synthesizer modules were applied as follows: $A(1,1$ cycle)-B-A(2, 1 cycle)-B-A(1, 2 cycles)-B-A(1, 1 cycle). The resulting tetrasaccharide was purified using normal phase HPLC (YMC Diol column). After methanolysis of the benzoyl esters the semi-protected tetrasaccharide was purified using normal phase HPLC (YMC Diol column) and subjected to hydrogenolysis, providing $6(3.4 \mathrm{mg})$ in $26 \%$ yield based on resin loading. ${ }^{1} \mathrm{H}$ NMR $\left(600 \mathrm{MHz}, \mathrm{D}_{2} \mathrm{O}\right): \delta 4.72(\mathrm{~d}, J=7.9 \mathrm{~Hz}, 1 \mathrm{H}), 4.49-4.39$ (m, 3H), 3.97-3.21 (m, 30H), $2.94(\mathrm{t}, J=7.5 \mathrm{~Hz}, 2 \mathrm{H}), 1.68-1.55(\mathrm{~m}, 4 \mathrm{H})$, 1.45-1.34 (m, 2H) ppm. ${ }^{13} \mathrm{C}$ NMR (151 MHz, $\left.\mathrm{D}_{2} \mathrm{O}\right): \delta 100.8,100.6,100.3$, 82.1, 76.8, 74.2, 73.7, 73.0, 72.6, 72.4, 71.4, 71.3, 71.2, 68.3, 67.7, 66.2, 58.8, 58.3, 37.6, 26.4, 24.7, 20.3 ppm. ESI-HRMS: $\mathrm{m} / \mathrm{z}[\mathrm{M}+\mathrm{H}]^{+}$calcd. for $\mathrm{C}_{29} \mathrm{H}_{54} \mathrm{NO}_{21}: 752.3188$; found 752.3181 .

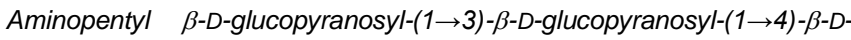

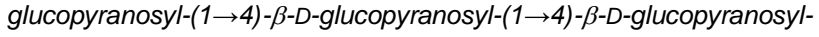
$(1 \rightarrow 3)$ - $\beta$-D-glucopyranoside (7)

The synthesizer modules were applied as follows: $A(2,1$ cycle)-B-A(1, 2 cycles)-B-A(1, 1 cycle)-B-A(1, 1 cycle)-B-A(2, 1 cycle)-B-A(1, 2 cycles)-B. The resulting hexasaccharide was purified using normal phase HPLC (YMC Diol column). After methanolysis of the benzoyl esters the semiprotected hexasaccharide was purified using normal phase HPLC (YMC Diol column) and purified again after hydrogenolysis using reversed phase HPLC (Hypercarb column), providing $7(2.1 \mathrm{mg})$ in $12 \%$ yield based on resin loading. ${ }^{1} \mathrm{H}$ NMR $\left(400 \mathrm{MHz}, \mathrm{D}_{2} \mathrm{O}\right): \delta 4.77-4.71(\mathrm{~m}, 2 \mathrm{H})$, 4.55-4.49 (m, 3H), $4.47(\mathrm{~d}, J=8.1 \mathrm{~Hz}, 1 \mathrm{H}), 4.01-3.29(\mathrm{~m}, 38 \mathrm{H}), 3.00-2.92$ $(\mathrm{m}, 2 \mathrm{H}), 1.71-1.60(\mathrm{~m}, 4 \mathrm{H}), 1.48-1.39(\mathrm{~m}, 2 \mathrm{H}) \mathrm{ppm} .{ }^{13} \mathrm{C} N M R(101 \mathrm{MHz}$, $\left.\mathrm{D}_{2} \mathrm{O}\right): \delta 100.6,100.4,100.2,99.7,82.2,81.6,76.2,76.1,76.0,73.8,73.3$ 72.6, 71.8, 71.2, 71.0, 70.7, 67.9, 67.4, 66.0, 65.8, 58.5, 58.3, 57.6, 37.2
26.0, 24.2, 19.9 ppm. ESI-HRMS: $\mathrm{m} / \mathrm{z}[\mathrm{M}+\mathrm{H}]^{+}$calcd. for $\mathrm{C}_{29} \mathrm{H}_{54} \mathrm{NO}_{21}$ 1076.4245; found 1076.4249 .

Aminopentyl $\quad \beta$-D-glucopyranosyl-(1 $\rightarrow 4)-\beta$-D-glucopyranosyl-(1 $\rightarrow 3)-\beta$-D-

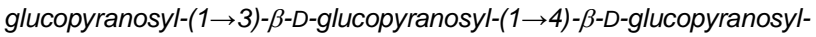
$(1 \rightarrow 4)-\beta$-D-glucopyranoside (8)

The synthesizer modules were applied as follows: $A(1,1$ cycle $)-B-A(1,1$ cycle)-B-A(2, 1 cycle)-B-A(2, 2 cycles)-B-A(1, 2 cycles)-B-A(1, 1 cycle)-B. The resulting hexasaccharide was purified using normal phase HPLC (YMC Diol column). After methanolysis of the benzoyl esters the semiprotected hexasaccharide was purified using normal phase HPLC (YMC Diol column) and subjected to hydrogenolysis, providing $8(2.2 \mathrm{mg})$ in $12 \%$ yield based on resin loading. ${ }^{1} \mathrm{H}$ NMR $\left(400 \mathrm{MHz}, \mathrm{D}_{2} \mathrm{O}\right): \delta 4.72(\mathrm{~d}, \mathrm{~J}$ $=8.0 \mathrm{~Hz}, 2 \mathrm{H}), 4.53-4.36(\mathrm{~m}, 4 \mathrm{H}), 4.02-3.18(\mathrm{~m}, 38 \mathrm{H}), 3.01-2.83(\mathrm{~m}, 2 \mathrm{H})$, 1.66-1.57 (m, 4H), 1.44-1.34 (q, 2H) ppm. ${ }^{13} \mathrm{C}$ NMR (101 MHz, D $\left.{ }_{2} \mathrm{O}\right): \delta$ 100.4, 100.1, 99.8, 81.7, 81.4, 76.3, 76.1, 73.8, 73.4, 73.2, 72.6, 72.1, 71.9, 71.1, 70.9, 70.7, 67.9, 67.2, 65.8, 65.7, 58.3, 57.7, 37.1, 26.0, 24.2, 19.9. ESI-HRMS: $\mathrm{m} / \mathrm{z}[\mathrm{M}+\mathrm{H}]^{+}$calcd. for $\mathrm{C}_{41} \mathrm{H}_{74} \mathrm{NO}_{31}$ : 1076.4245 ; found 1076.4240 .

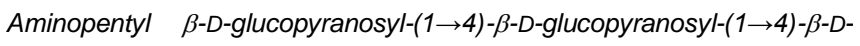

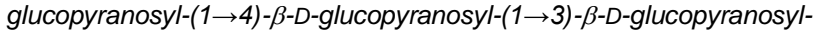
$(1 \rightarrow 4)-\beta$-D-glucopyranoside (9)

The synthesizer modules were applied as follows: $A(1,1$ cycle)-B-A(2, 1 cycle)-B-A(1x2 cycles)-B-A(1, 1 cycle)-B-A(1, 1 cycle)-B-A(1, 1 cycle)-B. The resulting hexasaccharide was purified using normal phase HPLC (YMC Diol column). After methanolysis of the benzoyl esters the semiprotected hexasaccharide was purified using normal phase HPLC (YMC Diol column) and subjected to hydrogenolysis, providing $9(6.5 \mathrm{mg})$ in $34 \%$ yield based on resin loading. ${ }^{1} \mathrm{H}$ NMR $\left(400 \mathrm{MHz}, \mathrm{D}_{2} \mathrm{O}\right): \delta 4.70(\mathrm{~d}, \mathrm{~J}$ $=7.9 \mathrm{~Hz}, 1 \mathrm{H}), 4.52-4.38(\mathrm{~m}, 5 \mathrm{H}), 3.98-3.16(\mathrm{~m}, 38 \mathrm{H}), 3.01-2.86(\mathrm{~m}, 1 \mathrm{H})$, 1.68-1.54 (m, 4H), 1.46-1.31 (m, 2H) ppm. ${ }^{13} \mathrm{C}$ NMR (101 MHz, $\left.\mathrm{D}_{2} \mathrm{O}\right): \delta$

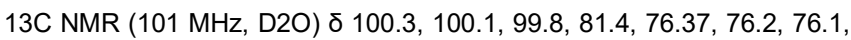
76.0, 73.8, 73.4, 73.2, 72.6, 72.1, 71.8, 71.7, 71.0, 70.9, 70.8, 70.7, 67.9, 67.2, 65.7, 58.3, 57.6, 37.1, 26.0, 24.2, 19.9 ppm. ESI-HRMS: m/z $[\mathrm{M}+\mathrm{H}]^{+}$calcd. for $\mathrm{C}_{41} \mathrm{H}_{74} \mathrm{NO}_{31}$ : 1076.4245; found 1076.4250 .

Aminopentyl $\beta$-D-glucopyranosyl-(1 $\rightarrow 4)-\beta$-D-glucopyranosyl-(1 $\rightarrow 4)-\beta$-D-

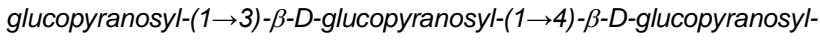
$(1 \rightarrow 3)$ - $\beta$-D-glucopyranoside (10)

The synthesizer modules were applied as follows: $A(2,1$ cycle)-B-A(1, 2 cycles)-B-A(2, 1 cycle)-B-A(1, 2 cycles)-B-A(1, 1 cycle)-B-A(1, 1 cycle)-B. The resulting hexasaccharide was purified using normal phase HPLC (YMC Diol column). After methanolysis of the benzoyl esters the semiprotected hexasaccharide was purified using normal phase HPLC (YMC Diol column) and subjected to hydrogenolysis, providing $10(3.5 \mathrm{mg})$ in $18 \%$ yield based on resin loading. ${ }^{1} \mathrm{H}$ NMR $\left(600 \mathrm{MHz}, \mathrm{D}_{2} \mathrm{O}\right): \delta 4.59-4.45$ (m, 4H), 4.05-3.26 (m, 38H), 3.02 (t, J= 7.6 Hz, 2H), 1.75-1.63 (m, 4H), 1.51-1.43 (m, 2H) ppm. ${ }^{13} \mathrm{C}$ NMR (151 MHz, $\left.\mathrm{D}_{2} \mathrm{O}\right): \delta 100.4,100.2,99.8$, 82.3, 81.6, 76.4, 76.3, 76.2, 73.8, 73.4, 73.3, 72.7, 71.9, 71.1, 71.0, 70.9, 70.8, 70.7, 67.9, 67.3, 66.0, 65.8, 58.6, 58.4, 57.8, 37.2, 26.0, 24.3, 19.9 ppm. ESI-HRMS: $\mathrm{m} / \mathrm{z}[\mathrm{M}+\mathrm{H}]^{+}$calcd. for $\mathrm{C}_{41} \mathrm{H}_{74} \mathrm{NO}_{31}$ : 1076.4245; found 1076.4213 . 


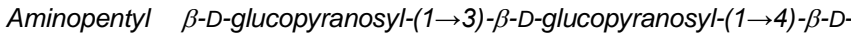

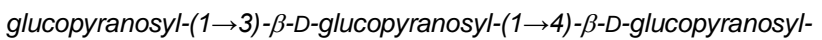
$(1 \rightarrow 4)$ - $\beta$-D-glucopyranosyl-(1 $\rightarrow 4)$ - $\beta$-D-glucopyranosyl-( $1 \rightarrow 3)-\beta$-Dglucopyranoside (11)

The synthesizer modules were applied as follows: $\mathrm{A}(2,1$ cycle)-B-A(1, 2 cycles)-B-A(1, 1 cycle)-B-A(1, 1 cycle)-B-A(2, 1 cycle)-B-A(1, 2 cycles)$B-A(2,1$ cycle)-B-A(1, 2 cycles)-B. The resulting octasaccharide was purified using normal phase HPLC (YMC Diol column). After methanolysis of the benzoyl esters the semi-protected hexasaccharide was purified using normal phase HPLC (YMC Diol column) and subjected to hydrogenolysis, providing $11(5.6 \mathrm{mg})$ in $23 \%$ yield based on resin loading. ${ }^{1} \mathrm{H}$ NMR $\left(600 \mathrm{MHz}, \mathrm{D}_{2} \mathrm{O}\right): \delta 4.59-4.52(\mathrm{~m}, 4 \mathrm{H}), 4.50(\mathrm{~d}, J=8.1$ $\mathrm{Hz}, 1 \mathrm{H}), 4.07-3.26(\mathrm{~m}, 50 \mathrm{H}), 3.02(\mathrm{t}, J=7.6 \mathrm{~Hz}, 2 \mathrm{H}), 1.75-1.65(\mathrm{~m}, 4 \mathrm{H})$, 1.50-1.44 (m, 2H) ppm. $\left.{ }^{13} \mathrm{C} \mathrm{NMR} \mathrm{(151} \mathrm{MHz,} \mathrm{D}_{2} \mathrm{O}\right): \delta 100.8,100.6,100.5$, 100.4, 99.9, 82.5, 82.0, 81.8, 76.6, 76.5, 76.4, 76.3, 74.0, 73.6, 73.6, 72.9, 72.8, 72.1, 71.4, 71.2, 71.0, 70.9, 70.9, 68.1, 67.6, 66.2, 66.0, 58.7, 58.6, 58.0, 57.9, 37.4, 26.2, 24.4, $20.1 \mathrm{ppm}$. ESI-HRMS: $\mathrm{m} / \mathrm{z}[\mathrm{M}+\mathrm{H}]+$ calcd. for $\mathrm{C}_{41} \mathrm{H}_{74} \mathrm{NO}_{31}$ : 1400.5310 ; found 1400.5301 .

\section{Analysis of glycosyl hydrolase substrate specificities}

Lichenase (endo-1,3:1,4- $\beta$-D-Glucanase) from Bacillus subtilis (GH16) was purchased from Megazyme (Bray, Ireland) and used in the following buffer that was suggested by the manufacturer: 100 $\mathrm{mM} \mathrm{NaPO}, \mathrm{pH}$ 6.5. The enzyme was used at a concentration of 1 $\mathrm{U} / \mathrm{ml}$ for the end-point measurements and at $0.33 \mathrm{U} / \mathrm{ml}$ for the timecourse experiments. The oligosaccharides were used at a concentration of $1 \mathrm{mM}$. All reactions were carried out at $40^{\circ} \mathrm{C}$ and terminated by incubation at $80^{\circ} \mathrm{C}$ for $5 \mathrm{~min}$. The reactions were analyzed on an Agilent 1200 Series HPLC equipped with an Agilent 6130 quadrupole mass spectrometer (MS) and an Agilent 1200 Evaporative Light Scattering Detector (ELSD). For the end point experiment the oligosaccharides were separated on a Hypercarb column (150 x $4.6 \mathrm{~mm}$, Thermo Scientific) using a water (including $0.1 \%$ formic acid)-acetonitrile (ACN) gradient at a flow-rate of 0.7 $\mathrm{ml} / \mathrm{min}$ starting at $2.5 \% \mathrm{ACN}$ for $5 \mathrm{~min}$, ramping up to $15 \% \mathrm{ACN}$ at 8 min, followed by a slow increase of $A C N$ to $30 \%$ at $40 \mathrm{~min}$, a steep ramp to $100 \% \mathrm{ACN}$ at $43.5 \mathrm{~min}$, a decline back to $2.5 \% \mathrm{ACN}$ from $46 \mathrm{~min}$ to $47 \mathrm{~min}$, and equilibration until $55 \mathrm{~min}$ at $2.5 \%$ ACN. For the time-course experiment the oligosaccharides were separated on a Synergi column $(150 \times 4.6 \mathrm{~mm}$, Phenomenex) using a water (including $0.1 \%$ formic acid)-acetonitrile ( $\mathrm{ACN}$ ) gradient at a flowrate of $1.0 \mathrm{ml} / \mathrm{min}$ starting at $0 \% \mathrm{ACN}$ for $5 \mathrm{~min}$, ramping up to $20 \%$ ACN at $20 \mathrm{~min}$, a steep ramp to $100 \% \mathrm{ACN}$ at $25 \mathrm{~min}$, flush of the column with $100 \% \mathrm{ACN}$ for $5 \mathrm{~min}$, a decline back to $0 \% \mathrm{ACN}$ from $30 \mathrm{~min}$ to $35 \mathrm{~min}$, and equilibration until $45 \mathrm{~min}$ at $0 \% \mathrm{ACN}$. The peaks in the ELSD traces were assigned based on their retention time and the corresponding masses in the MS.

\section{Acknowledgements}

We gratefully acknowledge financial support from the Max Planck Society, the German Research Foundation (DFG, Emmy Noether program PF850/1-1 to FP, SFB765), the Fonds der chemischen Industrie (Liebig-fellowship to FP) and an ERC Advanced Grant (AUTOHEPARIN to PHS). We acknowledge experimental contributions by H. S. Hahm.

Keywords: carbohydrates $\cdot$ automated glycan assembly $\bullet$ plant cell wall $\bullet$ mixed-linkage glucan $\bullet$ lichenase
R. A. Burton, G. B. Fincher, Molecular Plant 2009, 2, 873-882. M. J. Hill, Europ. J. Cancer Prev. 1997, 6, 219-225.

G. Frost, A. A. Leeds, C. J. Doré, S. Madeiros, S. Brading, A. Dornhorst, Lancet 1999, 353, 1045-1048.

C. S. Brennan, L. J. Cleary, J. Cereal Sci. 2005, 42, 1-13. a) S. Peat, W. J. Whelan, J. G. Roberts, J. Chem. Soc. 1957, 3916-3924; b) F. W. Parrish, A. S. Perlin, E. T. Reese, Can. J. Chem 1960, 2094-2104.

T. J. Simmons, D. Uhrin, T. Gregson, L. Murray, I. H. Sadler, S. C Fry, Phytochemistry 2013, 95, 322-332.

G. B. Fincher, Curr. Opin. Plant Biol 2009, 12, 140-147.

F. E. Elgharbi, A. Hmida-Sayari, M. Sahnoun, R.Kammoun, L. Jlaeil, H. Hassiri, S. Bejar, Carbohyd. Pol. 2013, 98, 967-975.

X. Xue, S. C. Fry, Ann. Bot. 2012, 109, 873-886.

N. Planas, BBA-Protein Struct. M. 2000, 1543, 361-382.

a) O. J. Plante, E. R. Palmacci, P. H. Seeberger, Science 2001, 291, 1523-1527; b) P. H. Seeberger, Acc. Chem. Res. 2015, 48, 1450-1463.

a) D. Schmidt, F. Schumacher, A. Geissner, P. H. Seeberger, F. Pfrengle, Chem. Eur. J. 2015, 21, 5709-5713; b) P Dallabernardina, F. Schumacher, P. H. Seeberger, F. Pfrengle. Org. Biom. Chem. 2016, 14, 309-313; c) M. P. Bartetzko, F. Schumacher, H. S. Hahm, P. H. Seeberger, F. Pfrengle, Org. Lett. 2015, 17, 4344-4347; d) M. P. Bartetzko, F. Schumacher, P. H. Seeberger, F. Pfrengle, J. Org. Chem., in press, DOI: 10.1021/acs.joc.6b02745.

M. W. Weishaupt, S. Matthies, P. H. Seeberger, Chem. Eur. J. 2013, 19, 12497-12503.

S. Eller, M. Collot, J. Yin, H. S. Hahm, P. H. Seeberger, Angew. Chem. Int. Ed. 2013, 52, 5858-5861.

N. M. McGregor, M. Morar, T. H. Fenger, P. Stogios, N. Lenfant, V. Yin, X. Xu, E. Evdokimova, H. Chui, B. Henrissat, A. Savchenko, H Brumer, J. Biol. Chem. 2016, 291, 1175-1197. 